\title{
Implant Treatment for the Geriatric Patient: Necessity or Desire?
}

\author{
Eric Z Shapira* \\ Professor of Geriatric Medicine and Dentistry
}

ISSN: 2637-7764

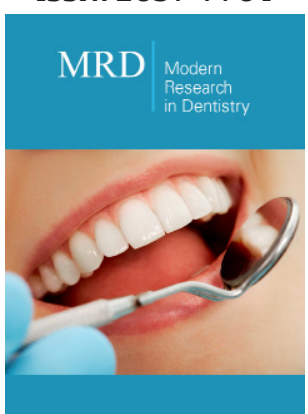

*Corresponding author: Eric Z Shapira, Professor of Geriatric Medicine and Dentistry, USA

Submission: 漈 February 17, 2020

Published: 㭗July 20, 2020

Volume 5 - Issue 3

How to cite this article: Eric $\mathrm{Z}$ Shapira. Implant Treatment for the Geriatric Patient: Necessity or Desire? An Improvement and Modification of Traditional GBR: A Report of Two Cases. Mod Res Dent. 5(3). MRD.000611. 2020. DOI: 10.31031/MRD.2020.05.000611

Copyright@ Eric Z Shapira, This article is distributed under the terms of the Creative Commons Attribution 4.0 International License, which permits unrestricted use and redistribution provided that the original author and source are credited.

\begin{abstract}
s
Today, people are living longer and hopefully keeping their teeth longer. There are modalities of dental treatment that can correct tooth loss and bone loss with adequate substitutes that emulate "the real thing." Bone loss, a disease symptom which can occur from multi-variants, can lead to subsequent tooth loss and an inability to replace these lost teeth. Dentists have long attempted to find ways of replacing missing teeth with endosseous, as well as subperiosteal implant techniques. Blade implants were the treatment of the 1950's; whereby a small length-wise slit was made on the edentulous ridge to a depth conducive to "tapping" a stainless steel, flat fixture into the bone. Depending upon the width and depth of the surrounding bone, the blade implant could be used to hold single and/or multiple crowns. Trial and error led to the advent of the cylindrical, square, peg-shaped or rounded and oblong implants made of titanium and other osseo-integrative materials.
\end{abstract}

Eventually, hydroxyl-appetite coated implants came into use and proved more successful than the standard blade type implant. The "coated" implants were more predictable and lasted longer, especially when coated with "plasma spray". Older individuals with more discretionary income, who may have been conditioned and committed to saving their teeth, were the more obvious patients opting for implant tooth replacement. Many factors that figured into the equation of whether the implant would "take" or not had to be considered before an implant could be delivered as the treatment of choice. Today, in conjunction with these various factors of viability and disease, considerations for recommending an implant as a replacement for a missing tooth should be given the highest and priority as it has become the Standard of Care.

\section{The Aging Physiology}

Aging brings change to everything. Therefore, the human body is subject to many stressors, including stress itself, disease, time, the normal aging process, and physical injury of one kind or another. All of these aforementioned entities potentiate change and either allows the human body to adapt physiologically or forcibly exerts this change unconsciously or consciously depending upon the type of insult one is trying to cope with at the time. The older one gets, the more difficult this process is and the slower the process becomes. Patients on multiple drug therapies, patients with various systemic disease states, patients with emotional stress, one Dementia of another, personality disorders and various metabolic problems should be examined thoroughly, both dentally and medically, prior to any implant placement. One should go as far as getting a Medical Clearance for an elderly, medically compromised patient to have the surgical placement of an implant. The human body is susceptible to rapid changes that may be slow to expose symptoms which may be indicative of subliminal disease states. Many diseases today can and should preclude one from having a dental implant due to the high risk of potential failure. Some of these disease states might include: osteopenia, osteoporosis, diabetes, auto-immune diseases, diseases related to material allergies, xerostomia, stressrelated bruxism, chronic advanced periodontal disease and the loss of mental cognition, which would limit eventual home care and recall visits post implant placement; ultimately leading to failure.

\section{Aging Issues}

This author believes that our greatest challenge as dental practitioners is to get the 
patient, especially the older patient, who needs implant therapy to want an implant procedure; and for that matter, anything else that demands a choice in restoring a patients' mouth to optimum health. That is: Our greatest challenge in dental practice is to get the patient to want what "we" think they need. All too often there remains a plethora of variables that can confound the choices we all make as patients. However, from experience, the biggest stopgap seems to be their abilities to afford implant therapy. There is no question in the mind of a person who for all their life has taken care of their mouth and the rest of their body, using self-efficacy as their guide, to want the best possible options to keep their dentition working in an ideal manner. There is no "hard sell" for the patient who knows what is best for themselves; but, for those patients who have difficulty making a decision for implant care, education seems to be at the core of this process. The question remains: Is it necessity, financial ability, rationalization through education or desire that helps in the process of getting to "yes?" Clinical examples can show how the prospective implant therapy for an elder can enhance their ability to function with embellished ability, a minimum of sideeffects and unnecessary angst about discomfort, ability to eat and other concerns (Table 1).

Table 1: A step-wise system of learning.

\section{A Mechanism for Communicating}

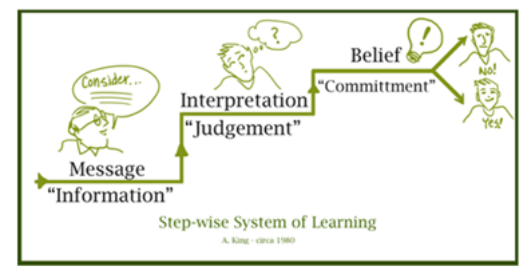

From Geriatric lectures of Eric Shapira, D.D.S. Avram King circa 1980's $($.

In the 1980's, a psychologist by the name of Avram [1] developed the Step-Wise System of Learning [1]. This table shows how one comes to making a decision given information that they did not have before. The persons' ability to choose is influenced by how the message is delivered of course, what kind of message it is e.g. didactive or guilt-inducing with judgment injected into the equation by the receiver. With the mixing of information, emotions, judgment and empathic or sympathetic words, a persons' level of commitment is born. The commitment can be getting to "yes" or ending in "no." This subtle graph is the crux of incentive-making tools that the dental practitioner should incorporate into his or her armamentarium. A patient who is resistant to learning or does not comprehend why they should have an implant procedure may be cognitively challenged, not speak the same language as the practitioner, or may be uneducated to the point of not understanding the whys and the wherefores of having to have an implant at all. The inability of the elderly patient choosing to have an implant may also be due to the inability of the dental practitioner themselves in explaining the need for the implant either because they do not do them directly, do not care to refer the patient out to a dentist who does, due to the fear generated about not getting the patient back, or the fact that the dentists' value system cannot justify anyone spending money on a treatment modality that they do not believe in themselves. According to an article in the Journal of the American Dental Association, Clark [2] has stated that "in the general population, the use of dental implants has become a management strategy for replacing missing teeth. As part of the treatment plan for the aging population, general dentists should consider this treatment modality in their practices. The rational delivery of this oral health care will assist in providing a high quality of life for these (older) patients [2]. Aging issues of concern include: systemic disease states, involved medical histories, complex drug therapies, complex social histories, genetic predisposition to periodontal disease, bone disease, allergies, and some specific emotional disorders or aberrant mental states.

\section{Implants-at-Large}

In 1949, Dr's Norman Goldberg and Aaron Gershkoff wrote an article published in Dental Digest that discussed their research and eventual success in coming up with techniques and designs for forming and placing dental implants after WWII [3]. They subsequently wrote the first textbook on Implant Dentistry in 1957. In the 1950's the blade implant came into its own for replacing missing teeth and was fabricated from stainless steel. This type of implant was used primarily where very thin widths of bone existed in the mandible or the maxilla as well as minimal depth of bone; however, their survival rate was limited due to lateral forces which ended up fracturing bone and the onset of infection, such as osteomyelitis, with adjacent bone loss. Later versions of this implant and a change in material to titanium helped it to come back into favor; however, the endosseous implants have taken the lead in today's dental marketplace.

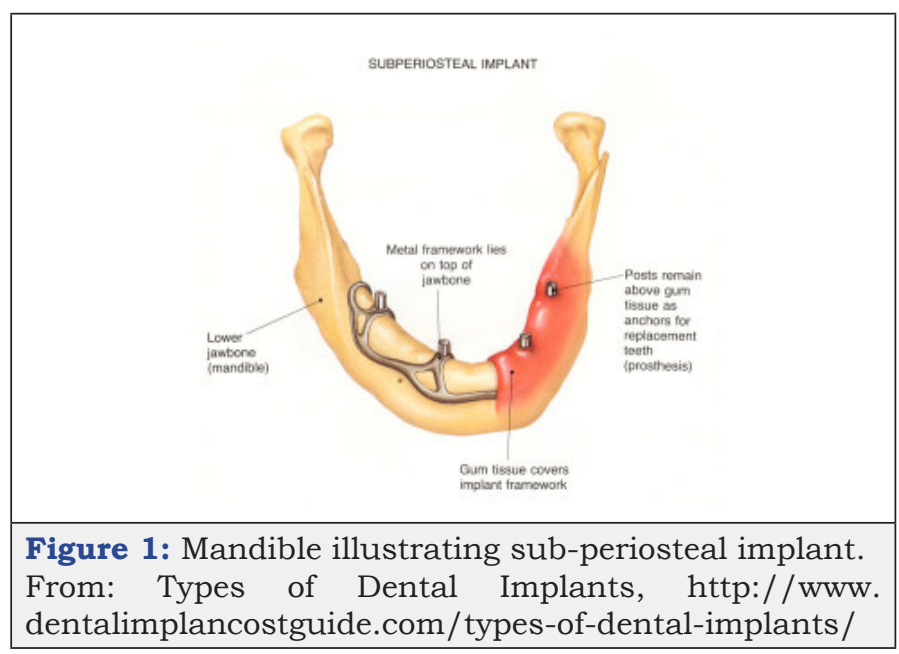

Today's dental implants can be categorized by their shape and placement as well as by the procedure used to place them, e.g. single or two-staged. The implant can be "buried" under the soft tissue or left above the tissue. If the implant is left under the tissue as a first stage, the second stage would be to uncover it by means of a scalpel or laser, remove the healing screw and place a healing cuff of specific height above the gingiva that will, in time, contour the gingiva with a flare or specific "immersion profile," while healing. 
The final stage would be to place the abutment head and take an impression for the subsequent crown. The following Figures 1-4 [4] illustrate types of implants, nomenclature of implants based on materials and surface coating as well as placement of the different implants.
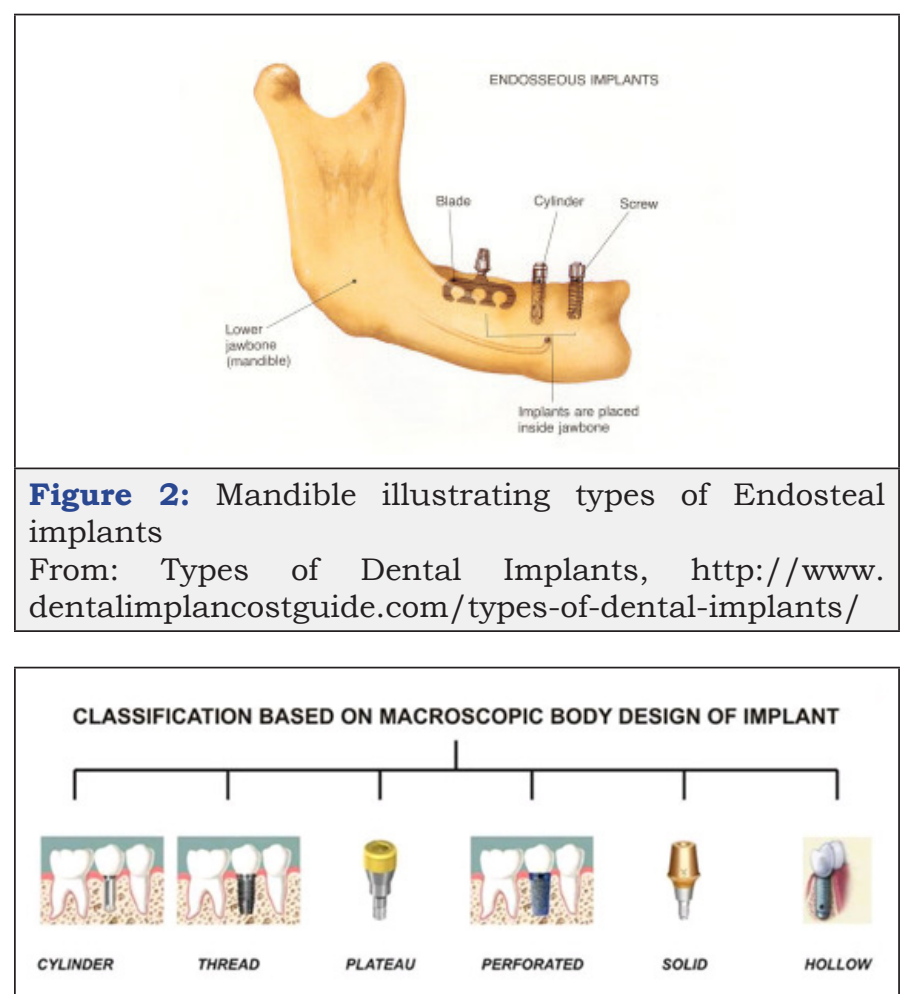

Figure 3: Types of body designs.

From: Types of Dental Implants, http://www. dentalimplancostguide.com/types-of-dental-implants /

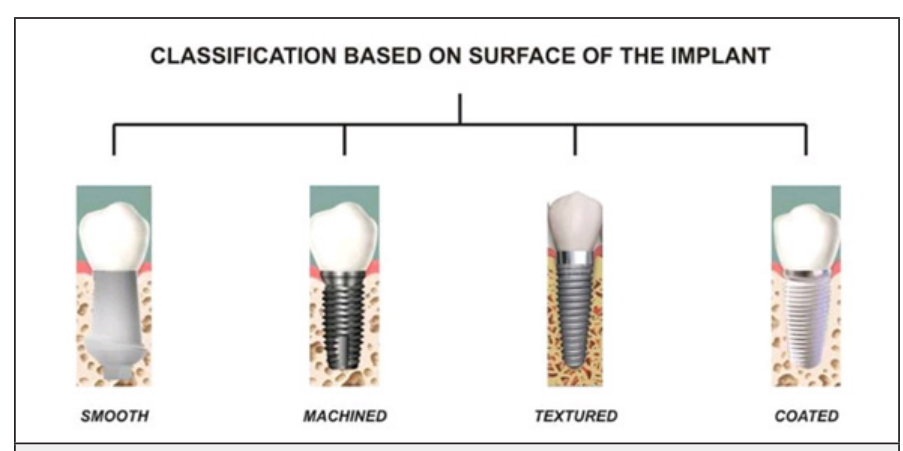

Figure 4: Types of Implant surfaces.

From: Types of Dental Implants, http://www. dentalimplancostguide.com/types-of-dental-implants /

Sub-periosteal implants have been commonly used for single and two stage procedures in the past. They are positioned inside the gum tissue on the jawbone. The post protrudes from the gums to hold the restoration. The primary advantage of this type of implant is that it can hold dentures securely for individuals who do not have enough bone height. This procedure can be accomplished in a two-stage surgical procedure; one exposing the bone and taking an impression and two, exposing the bone a second time and placing the implant under the periosteum after it has been fabricated by a dental laboratory. More modern technology allows for a CAT scan (Computerized Axial Tomography) of the jawbone, with a resultant three-dimensional model that can be used for fabricating the implant itself. Thus, a one stage placement can be made reducing the stress of having two surgeries, discomfort, and inconvenience. Older individuals who opt for this procedure should be limited to the one stage technique, if possible, due to fragility and deleterious side-effects. The Endosteal dental implants are the most used implants today, especially for two-stage procedures. They are a good alternative to bridges or detachable dentures; however, they can be used to support over-dentures in a very effective way using implant bar support, locator attachment's as well as a large number of other attachments. These implants come in several types including screw/threaded, cylinder/smooth and bladed. The cylindrical and tapered screw implants are usually coated and/or acid-etched for better osseous integration. These implants come in mini and standard sized diameters and lengths.

\section{Types of Surface Treatments}

The vast majority of dental implants are made of titanium, but the surface material of the implant varies. The material used is a major factor in the treatment's stability and long-term strength. Types of implant surface treatments are acid-etched, grit-blasted forming a roughened surface, plasma-sprayed hydroxyl-apatite coating, and a plasma-sprayed or microgroove titanium surface.

\section{Connectors}

Dental implants are also categorized by the types of abutment heads that are used. The abutments may be cemented or screwed into position. The design of the abutment can encompass an internal hex, an external hex or an internal octagon connector which has an opening in the dental implant head into which the abutment is screwed for restoration and possible eventual removal. Connectors can be manufactured from various metals and materials such as gold, titanium, zirconium, and porcelain.

\section{Bone health and geriatric individuals}

Bones consist of cells embedded in a mineralized matrix with collagen fibers. The primary tissue of bone is made mostly of calcium phosphate or calcium hydroxyl-apatite. The outer surface of the bone, especially of the face and jaw, is made up of cortical or hard bone. The internal surface of the bone is soft medullary bone or cancellous bone. Both the inferior border of the mandible and the superior surface of the mandible are formed of dense cortical bone. However, when teeth are lost, this bone disintegrates, and the height of the mandible is lowered as in older individuals who have lost their teeth later in life (Figures 5 \& 6) [5].

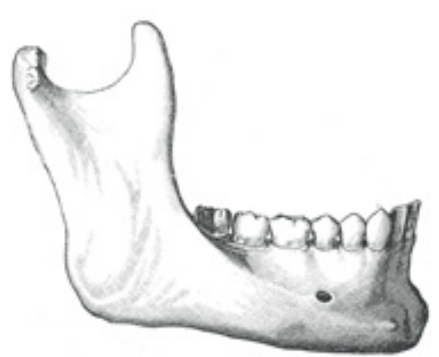

Figure 5: Adult mandible with teeth. From: Gray's Anatomy. 


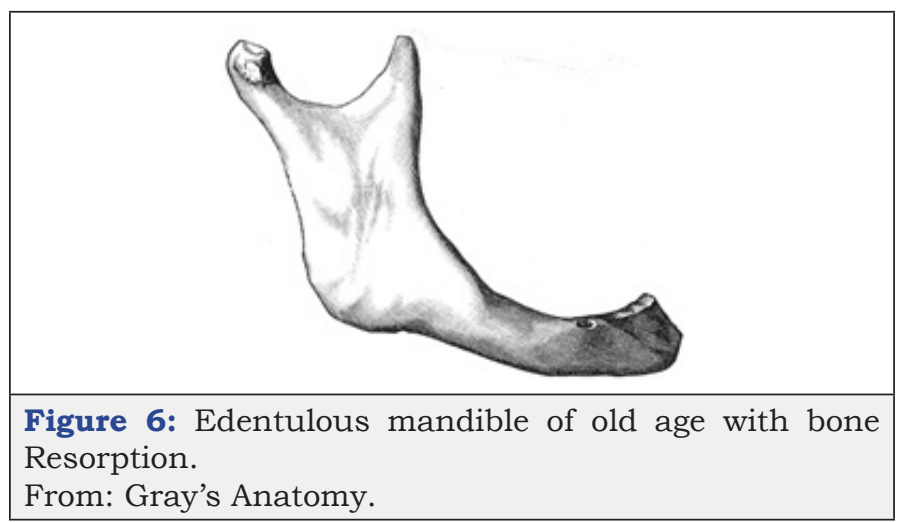

\section{From gray's anatomy}

Without the appropriate bone, both in height and width, implant therapy for most patients is limited. The older one gets, the more prone he or she is to diminished bone presence in one form or another. The mandible is a good indicator of bone loss and with associated tooth loss, bone loss is accelerated.

\section{Osteopenia and osteoporosis}

Osteopenia is a condition where bone mineral density is low, but not considered low enough to be labeled Osteoporosis. Osteopenia is one condition where what we do and how we act has an enormous effect on our improvement or lack thereof. There is no one single cause of this condition. Some of the known causes of osteopenia are the following:
A. Chronic dehydration
B. Genetics and age
C. Sedentary lifestyle
D. Stress
E. Dieting
F. Alcohol use
G. Caffeine
H. Sodium
I. Tobacco use, Secondhand smoke and Exposure to Cadmium

J. Excessive exercise

K. Irregular eating patterns and food choices

L. Phosphorous imbalance- too much or too little

M. Too much protein

Osteoporosis is a definite low mineral bone density which leads to fractures and "weak" bones. It is found in a higher percentage of older women, but men are not immune to this disease. Family history plays an important role in this disease as it is linked through hereditary factors, which increases ones' risk to excessive bone loss, fragility fractures and overt fractures, especially fractured hips with associated falls in the elderly. Approximately three fourths of the deaths that are due to falls in the United States occurring in
$13 \%$ of the population that is aged 65 and older. The estimated $1 \%$ of fallers who sustain a hip fracture have a $20 \%$ to $30 \%$ mortality within 1 year of the fracture. Fractured hip being the number one killer in people over the age of 65 who fall and find themselves with this condition [6]. Risk factors in osteoporosis include the following:

\section{A. Heredity}

B. Ethnicity (Caucasian and Asian women, then Hispanic. African American women have the lowest risk).

C. Low body weight (Body Mass Index of less than 19).

D. Menstrual history (If first menstruation is at 15 years of older, there is a greater risk of osteopenia and osteoporosis).

E. Lifestyle: Sedentary; Dieter; Cigarette smoking; Alcohol use.

F. Nutrition and Diet

G. Diseases creating risk factors: Intestinal disorders; Gastrectomy; Hyperthyroidism; Hyperparathyroidism; Multiple Sclerosis; Thalassemia: Severe liver disease; Multiple Myeloma; Type I Diabetes; Lactose Intolerance

H. Treatment for: Chronic inflammatory disease; Rheumatoid arthritis; Leukemia and lymphoma; Endometriosis

I. Drugs: Glucocorticoids; Aluminum containing drugs; Anticonvulsants; Cytotoxic drugs; Heparin; Lithium; Excessive thyroid hormone replacement; Diuretics other than Thiazide; Long course of antibiotics.

Osteoporosis can impede the longevity of our dentition and can be a detractor from having dental implant therapy. The success of osseointegration depends upon, in part, the host bed. Meaning, if a question about osteoporosis has been raised regarding a preexisting condition with decreased bone quantity and quality, how will this affect the overall longevity and placement of a dental implant? Osteoporosis is a definite risk factor for osseointegration [7]. Apparently, definitions of osteoporosis that site reduced bone density or fragility fracture may not be synonymous. That is, a patient who is diagnosed as osteoporotic does not necessarily have an "abnormal" amount or quantity of bone either in the jaws or in the other parts of the skeleton. This disease is primarily diagnosed in the presence of clinical manifestations, such as bone fracture due to a subsequent fall or possibly rapid onset periodontal bone loss. One must consider a Dexa scan, ${ }^{\mathrm{T}}$ in order to achieve evidence of low bone density with a subsequent diagnosis of osteoporosis. Dexa scans are the most used test to measure bone density [8]. A literature review does point out that osteoporosis may not be a risk factor for osseointegration of dental implants. However, one must be prudent in doing an optimum examination of the soft and hard tissue where an implant may be placed.

\section{CBCT}

Cone Beam Computerized Tomography (CBCT) is a necessary adjunct for the dental practitioner in being able to see the bone and surrounding anatomy present in a three-dimensional aspect. This 
allows for better and safer pre-treatment planning of a projected dental implant. No patient, especially geriatric patients, should have an implant placed without first using this modality. The accompanying CBCT [9] (Figure 7) of one of this author's older patients shows a graded scale in the top center portion of the picture. The author intends to replace a maxillary second molar. The dark area above the first molar is the maxillary sinus with approximately $4 \mathrm{~mm}$ of bone present on the distal aspect. This is the minimal amount of bone to do a crestal sinus approach. In actuality, on preparation with a crestal approach for a sinus lift, there was approximately $10 \mathrm{~mm}$ of bone present and an $11.5 \mathrm{~mm}$ implant was placed into the sinus and with bone and without perforation.

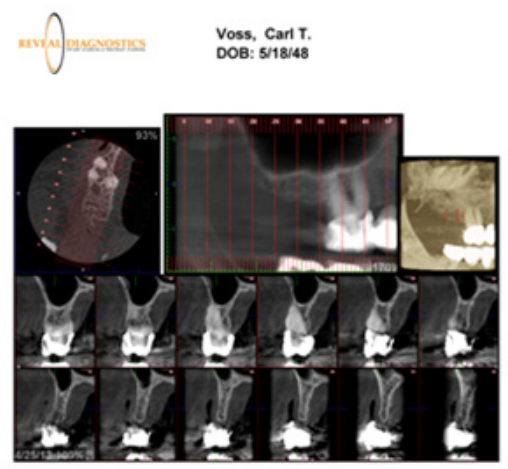

Figure 7: $\mathrm{CBCT}$ of the right maxilla area prior to implant placement for tooth \#2.

From: Patient records of Eric Shapira DDS with permission from C. Voss. (C).

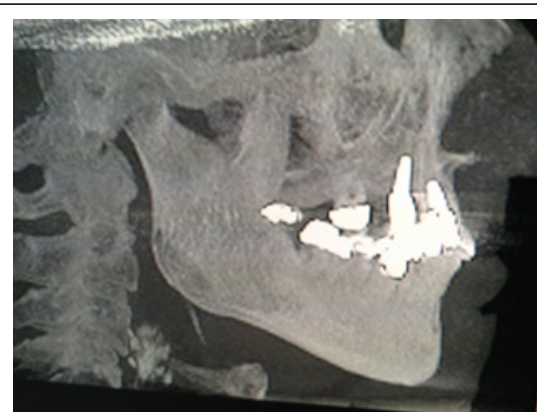

Figure 8: Radiograph showing implant placement into maxillary sinus with bone graft.

From: Patient records of Eric Shapira DDS used with permission $($ )

A photo of a similar crestal approach sinus lift in the maxillary right quadrant is seen with an implant placement (Figure 8) [10]. On the CBCT scan, a corresponding number will allow the practitioner to look at a tomographic side view of the area to be grafted. The cross-sectional pictures allow one to see the width and direction of the bone. Today with "Guided" Implant Surgery, the procedure is effortless compared to the past. A surgical stent is made utilizing the 3-D CBCT on six axis's and all the surgery is done through the stent that guides the drill of the practitioner to the proper length and direction the implant should be placed with respect to the local anatomy around it. This procedure is virtually painless and heals in short-order.

\section{The risk of bisphosphonates and smoking on implants in geriatric patients}

Patients, especially older patients, taking bisphosphonate therapy for the prevention of osteoporosis need to be aware of the side-effects of this drug with respect to the bone itself and the risks associated with using this drug. Recent knowledge from ongoing clinical research has shown that the use of bisphosphonates has been associated with causing osteonecrosis of the jaw, especially when used intravenously [11]. One of every two women will sustain an osteoporosis-related fracture in various locations about the body in her lifetime. More than 10 million Americans older than 50 years of age have osteoporosis, while another 34 million are at risk [12]. With respect to placing implants in patients who have osteoporosis or osteopenia, it is incumbent upon the practitioner to make a judgment call about the health and the availability of proper bone for implant placement. Smoking, especially long-term smoking in older individuals who are seeking out implant therapy is contraindicated for treatment. Studies have shown that smokers have a higher rate of implant failure [13].

\section{Dental implants and the geriatric patient}

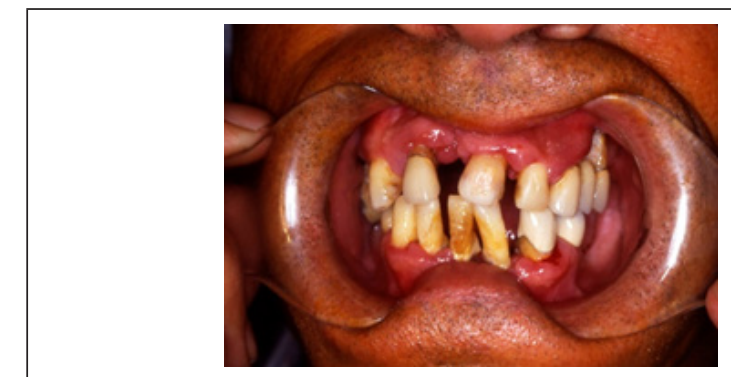

Figure 9: 75 yr. old for pre-implant consult $\mathbb{C}$ From: Deceased patient records of Eric Shapira, DDS.

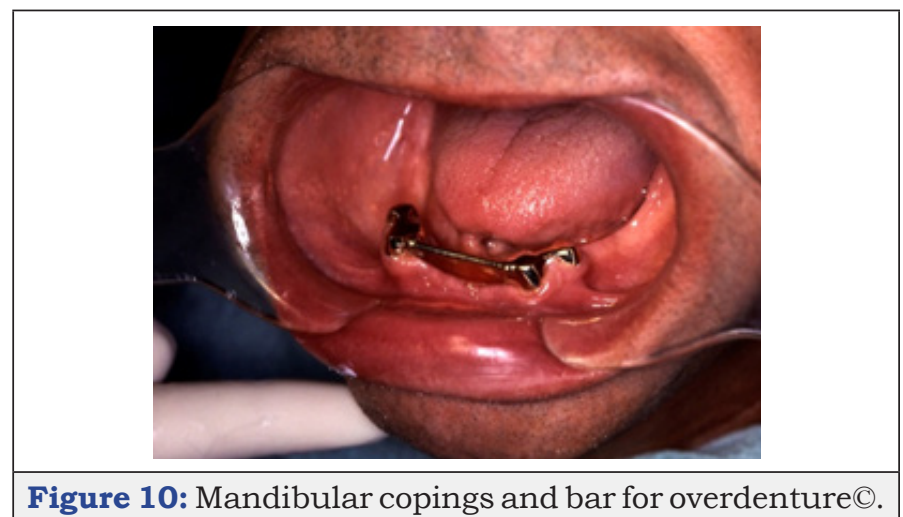

As previously stated, our greatest challenge as practitioners is to get our patients to want what we think they need. However, we need to know our patient's. Most aging patients are in good health from this authors' experience. It is not until one is limited in activity or subject to a systemic or mental disease that the patient may not make the choice to have an implant when it will clearly help them function in improved health. The patient who is motivated to do anything it takes to keep their mouth healthy, is a potential candidate for implants. A patient presented to this authors' office seeking implant treatment. I found the patient to be both medically 
compromised and with a low dental IQ. He had chronic periodontal disease, mobility of teeth, and generalized poor oral care.

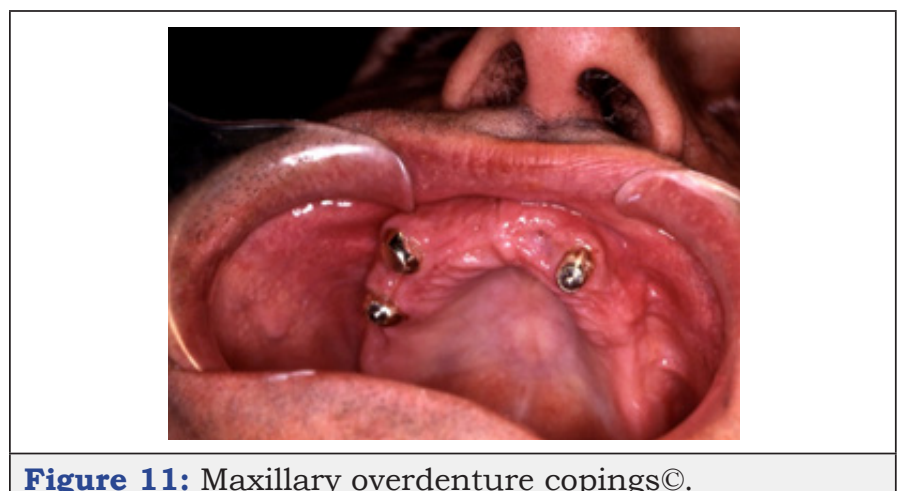

Figure 11: Maxillary overdenture copings (C.

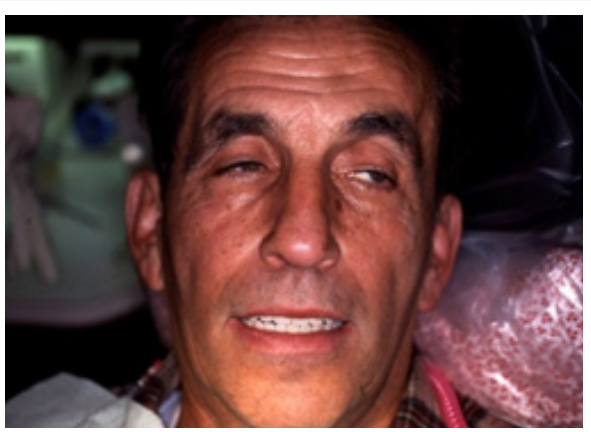

Figure 12: Completed maxillary and mandibular overdentures $($ ).

He was told that if he could maintain his mouth in an optimum condition with an alternative treatment, a set of overdentures, then we would consider implant therapy with fixed restorative in the future (Figures 9-12). After a year, he was so comfortable with his overdentures; he decided he did not need implants. It is not always necessary to oblige patients who think they want implants but do not really have the judgment to know why they should not have them. There must be a lot of rapport and trust between the patient and dentist in order to get to "yes." The patient has the right to know the options, the limitations, the risks, and the costs associated with dental implants and all other alternatives. Wants and desires are necessary in order to get to "yes" and needs are basic, but must be explained in depth with examples, models, and referrals to patients who have had similar treatment. This creates trust between the patient and dentist. Without trust, there is no dentist-patient relationship. The older individual depends upon your care, communication, and concern in assisting them with their overall oral health needs; especially making a choice about implant therapy.

\section{References}

1. King A (2013) A step-wise system of learning 1980's.

2. Stanford CM (2007) Dental implants: A role in geriatric dentistry for the general practice? JADA 138 Suppl: 34S-40S.

3. (2013) American Academy of Implant Dentistry. Chicago, Illinois, USA.

4. http://www.dentalimplantcostguide.com/types-of-dental-implants/

5. Drake R, Vogl WA, Mitchell AW (2009) Gray's Anatomy. Livingstone Publication, England.

6. Rubenstein LZ, Josephson KR (2006) Falls and their prevention in elderly people: what does the evidence show? Med Clin N Am 90(5): 807-824.

7. Dao TTT, Andersen JD, Zarb GA (1993) Is osteoporosis a risk factor for osseointegration of dental implants? Int J Oral Maxillofac Implants 8(2): 137-144.

8. http://www.osteopenic3.com/dexa-scans.html.

9. CBCT Scan from Author's records used with permission.

10. CBCT from Author's records used with permission.

11. American Dental Association Council on Scientific Affairs (2006) Dental management of patients receiving oral bisphosphonate therapy: Expert panel recommendations. JADA 137(8): 1144-1150.

12. Office of the Surgeon General (US) (2004) Bone health and osteoporosis: a report of the surgeon general.

13. Esposito M, Hirsch JM, Lekholm U, Thomson P (1998) Biological factors contributing to failures of osseointegrated oral implants. (II). Etiopathogenesis. Eur J Oral Sci 106(3): 721-764. 